\title{
Synthesis of Piperidines Using the aza-Prins Cyclization of n-Aryl Vinylogous Sulfonamides
}

\section{Dillon P. Cao}

ACS Spring 2021 National Meeting

April 12, 2021

Donahue Research Group

School of Mathematics and Natural Sciences

University of Southern Mississippi

Hattiesburg, MS 39406 


\section{Biography}

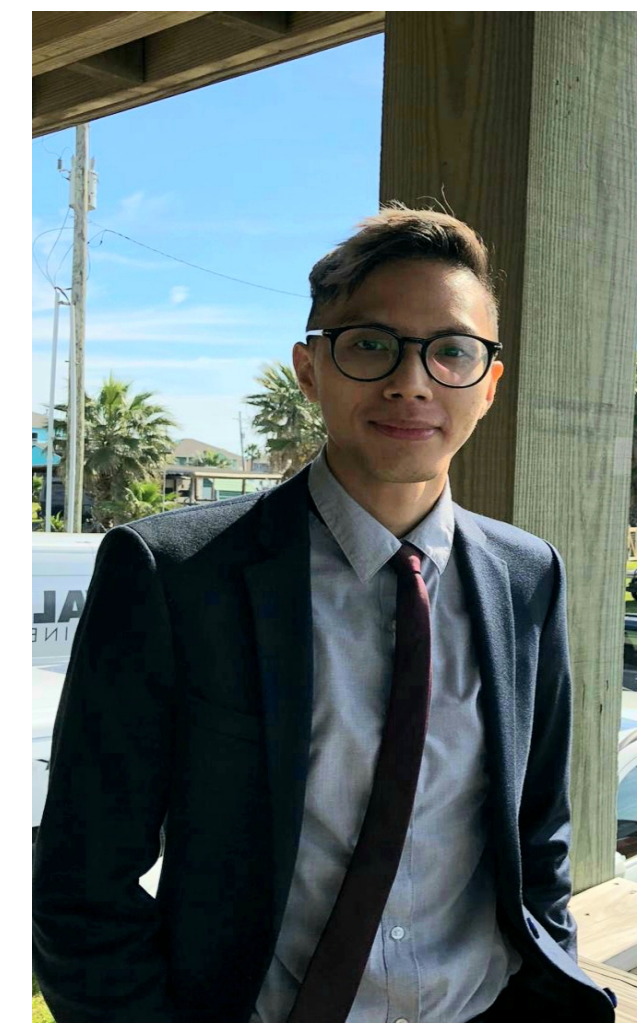

Dillon P. Cao
Born and raised in Biloxi, MS

2020: B.S in Chemistry (biochemistry emphasis) University of Southern Mississippi; Hattiesburg, MS Donahue Research Group Research: Investigation of acid-catalyzed cyclizations of $n$-sulfonyliminium ions to synthesize piperidine derivatives

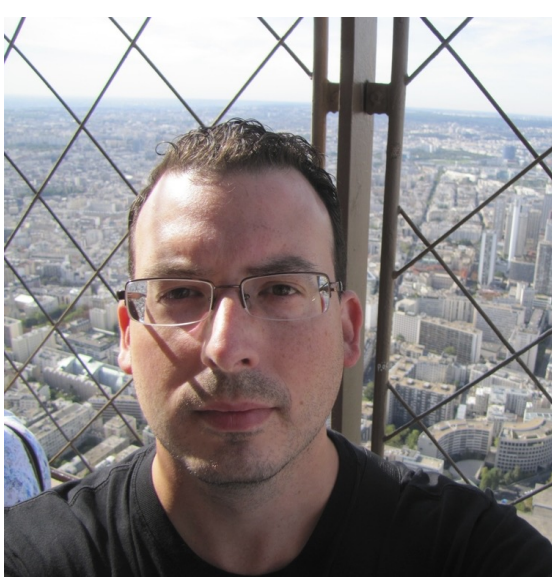

Matthew Donahue, PhD.

2020-Current: Finishing first year at Louisiana State University's PhD. Program specializing in organic synthesis Rivas Research Group Research: Implementing organic methodology and chemical biology to understand mode of action in biological systems with the goal of identifying novel therapeutic compounds.

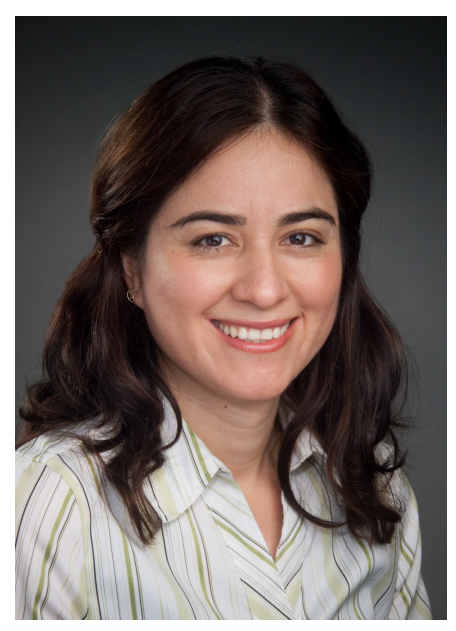

Fatima Rivas, PhD. 


\section{Background}
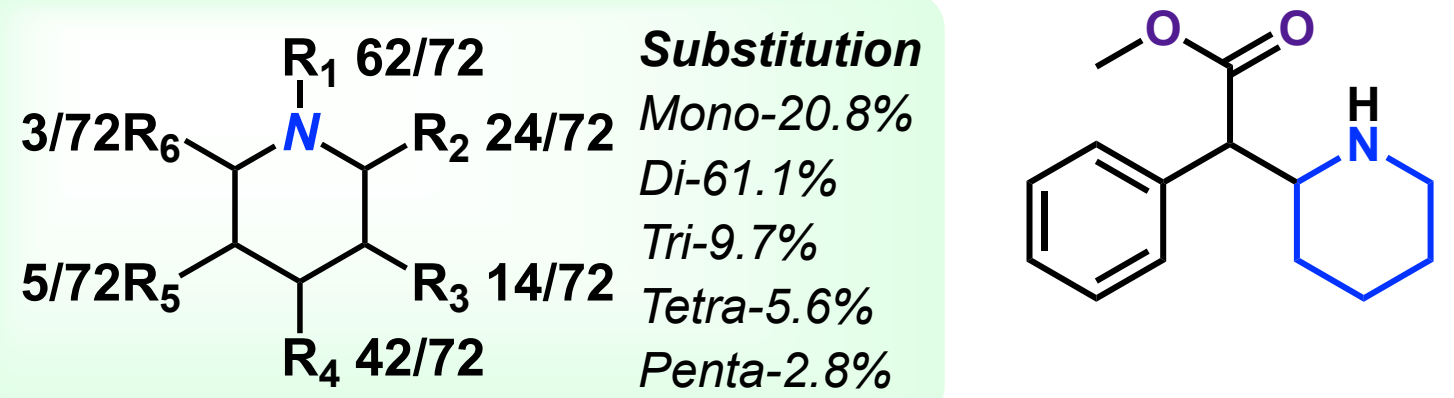

Methylphenidate

Trade Name: Concerta, Ritalin

Stimulant medication used to treat ADHD and narcolepsy

Acid-Promoted Prins Cyclizations of Enol Ethers to Form Tetrahydropyrans

David J. Hart and Chad E. Bennett, Org. Lett. 2003, 5 (9), 1499-1502
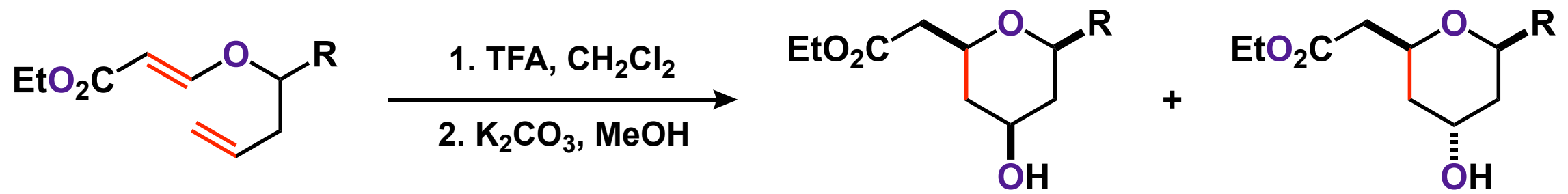

Acid-Promoted aza-Prins Cyclizations of Vinologous N-Sulfonyl Carbamates to Form

Piperidines
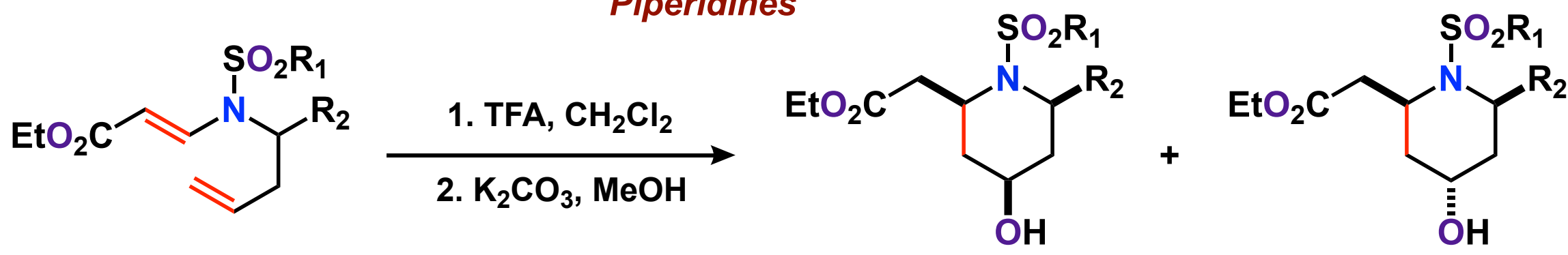

- - - - - - - - - - - - - - - - - - - - - - - - - - - - - 


\section{Scope of Study}

The $N$-Sulfonyliminium ion provides a powerful electrophile in which electrons can be accepted to encourage cyclization yielding a piperidine.

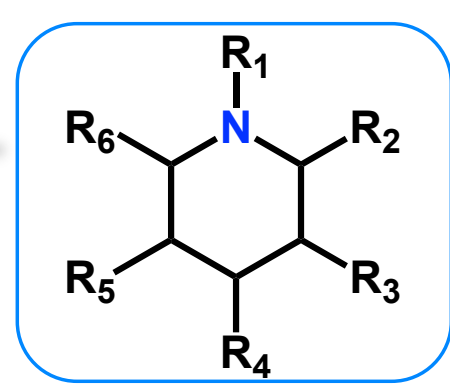

Hypothesis: The ambiphilic nature of $\mathrm{N}$-Vinolygous sulfonamides will be sufficiently basic to be activated by organic acids to generate an N-sulfonyliminium ion which will then trigger the intramolecular cyclization. The resulting $\boldsymbol{C}_{\mathbf{4}}$ carbocation can undergo stereoselective nucleophilic attack to yield the respective piperidine derivatives.
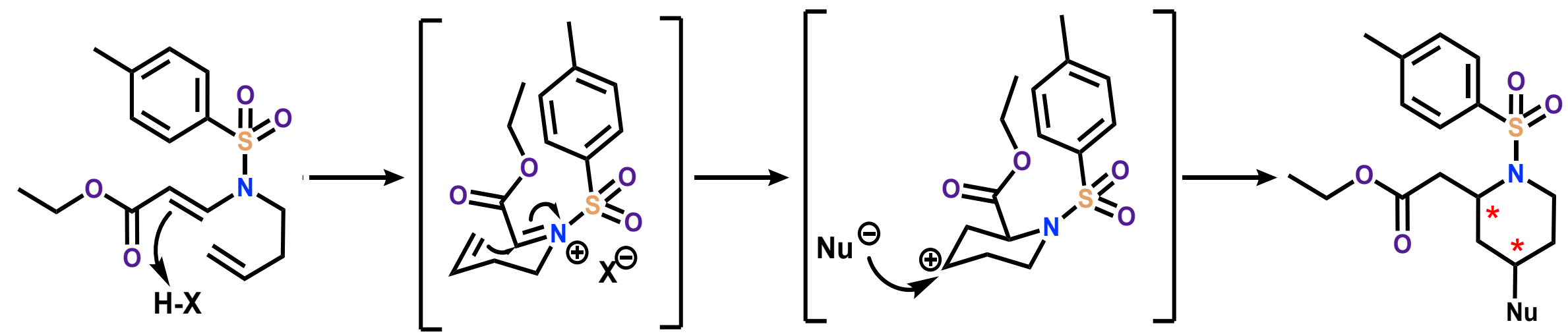

$\mathrm{N}$-Sulfonyliminium ion 


\section{Retrosynthetic Analysis}
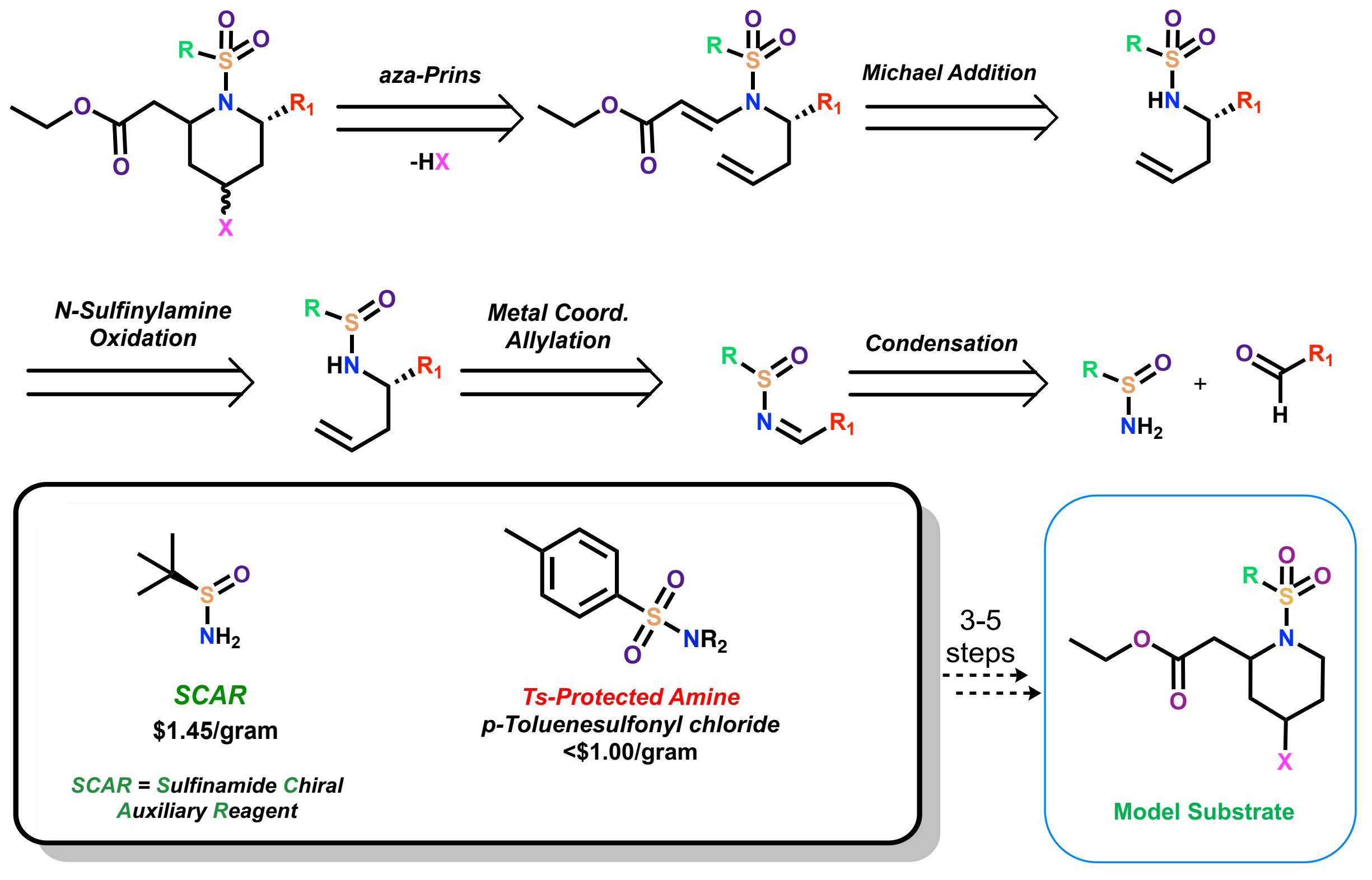


\section{Model Substrate}
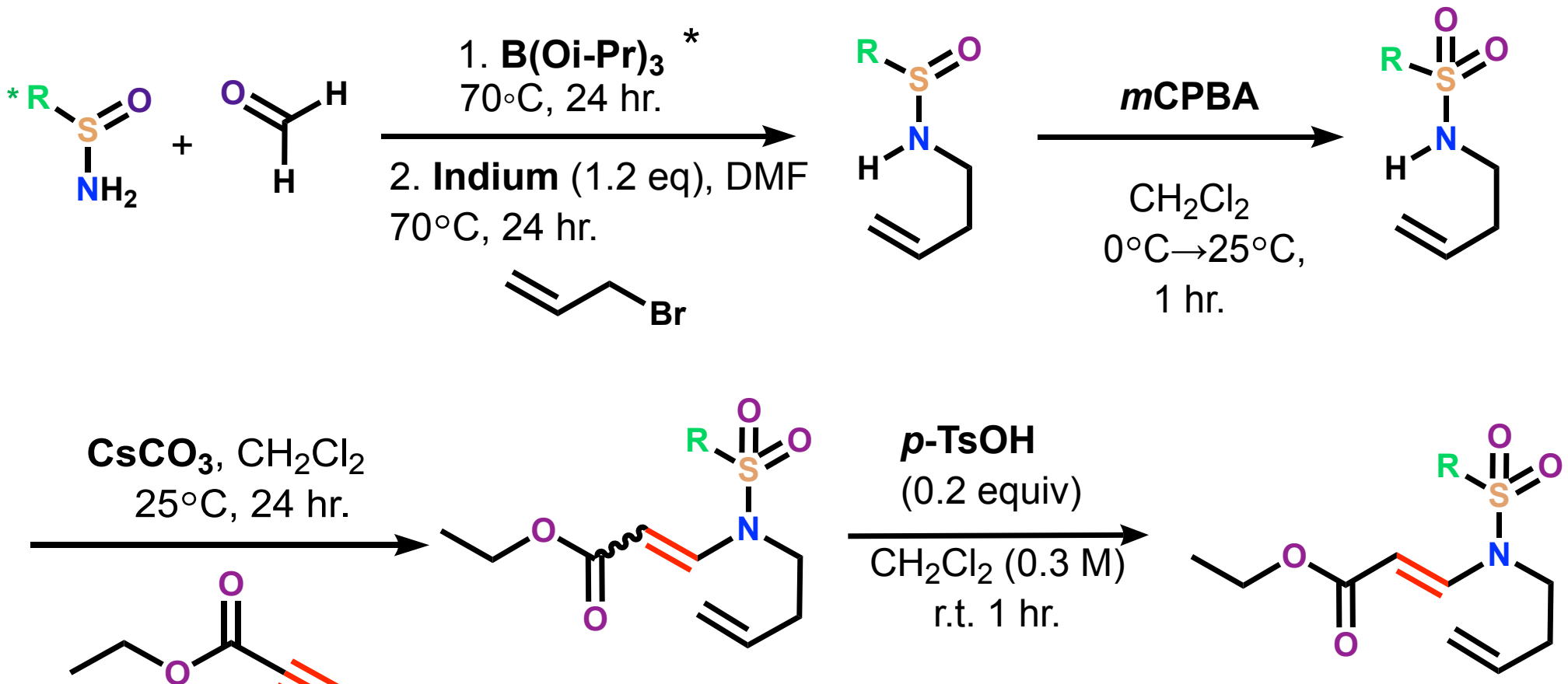

E:Z

2:1

$90 \%$

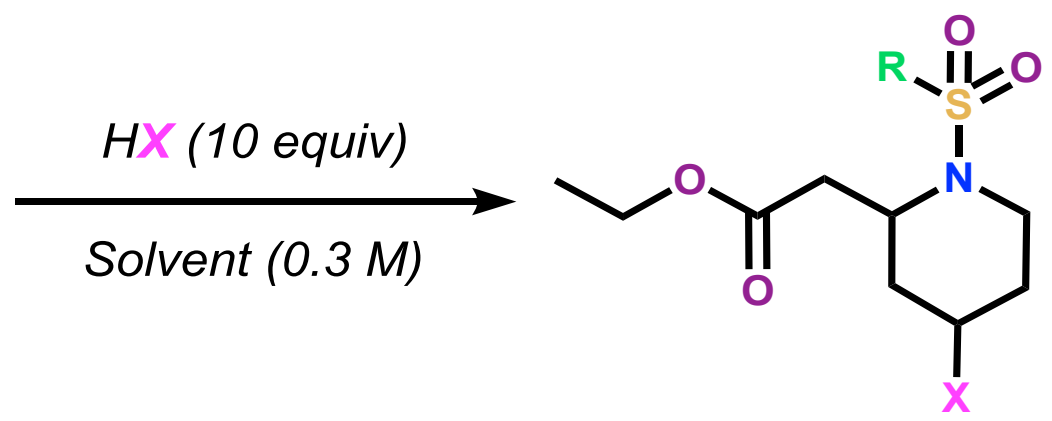

${ }^{*} \mathrm{R}=\boldsymbol{p}$-Tolyl, $\boldsymbol{t}$-Butyl 


\section{Michael Addition Base Screen}

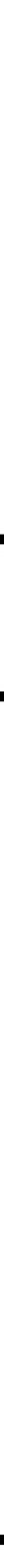

*Andna, L. Org. Biomol. Chem., 2019, 17, 5688-5692. 


\section{Key NMR Intermediates}

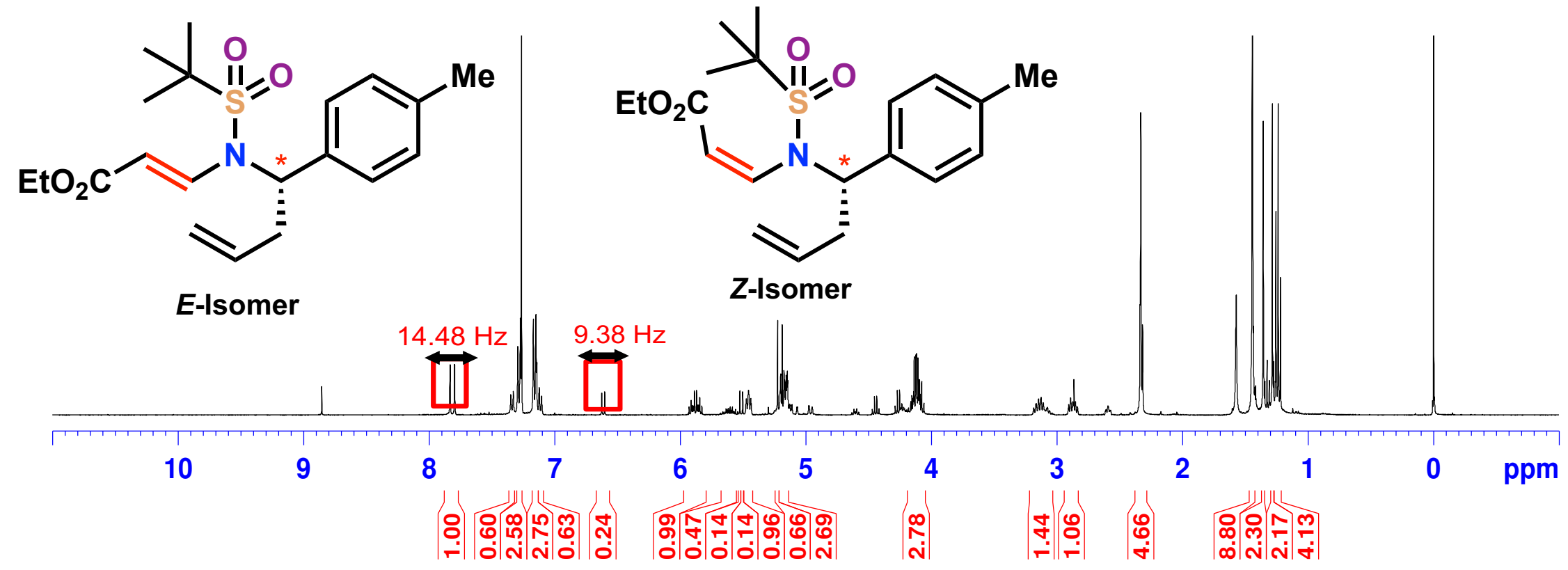

Isomerization to $E$-Isomer

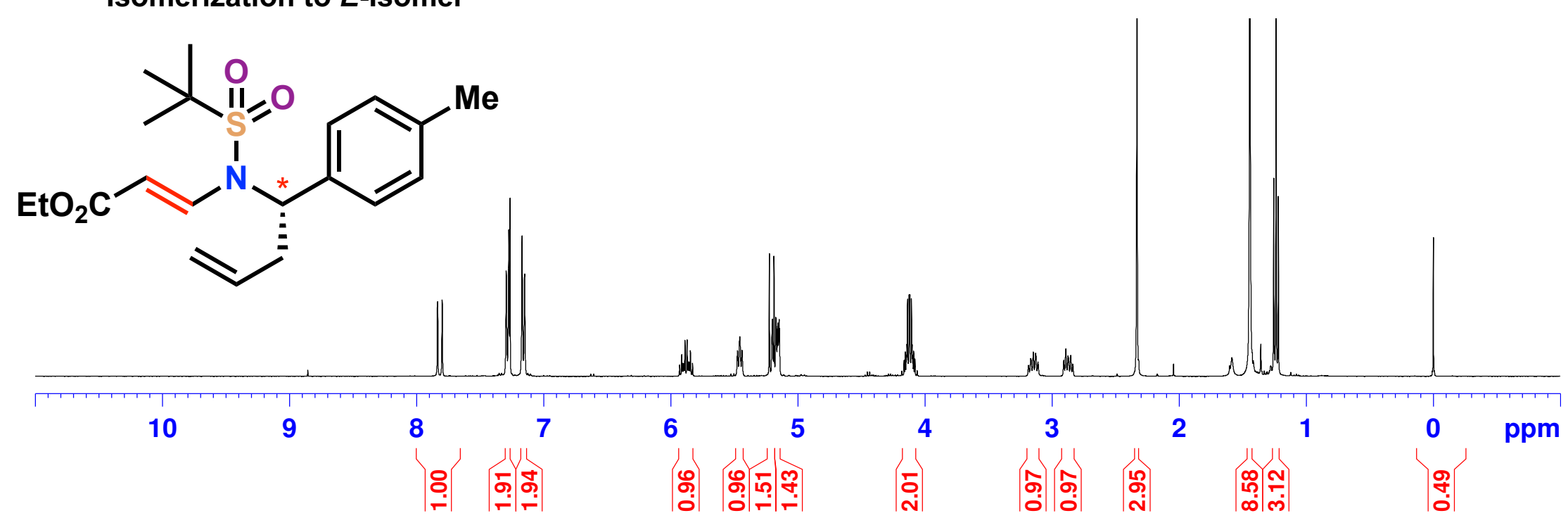




\section{aza-Prins Acid Screen (Current)}

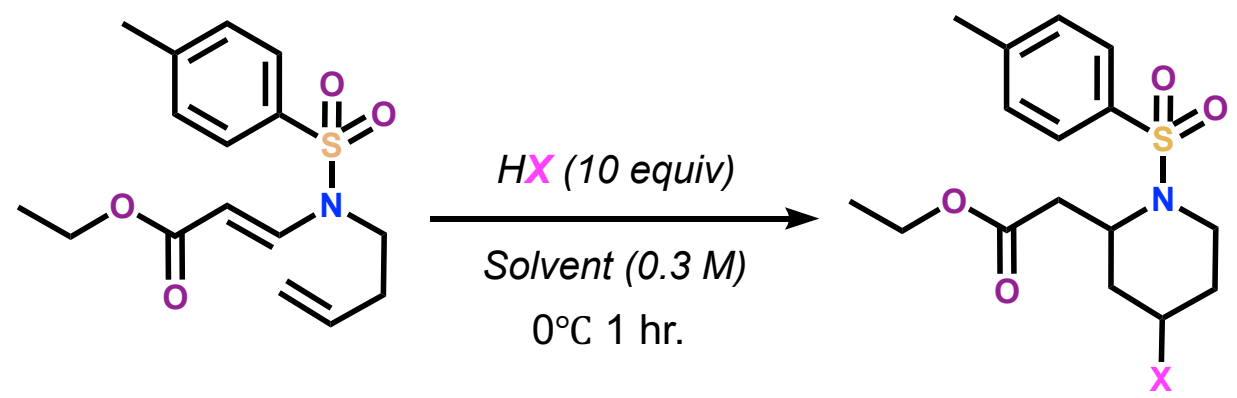

formic acid (3.77)<smiles>O=CO</smiles><smiles>Cc1ccc([As](=O)(=O)O)cc1</smiles><smiles>C=CC[S@](=C)N1CCC(OC=O)CC1CC(=O)OCC</smiles>

\section{Cyclized Piperidine Derivative Library}

methanesulfonic acid( (-2.6) trifluroacetic acid( (-0.26)<smiles>CS(=O)(=O)O</smiles><smiles>O=C(O)C(F)(F)F</smiles><smiles>O=S(=O)(c1ccc(I)cc1)c1ccc(I)cc1</smiles><smiles>CCOC(=O)CC1CC(OS(C)(=O)=O)CCN1C</smiles>

Bistriflamide ( $<-2.66)$

$$
\begin{aligned}
& \text { O } \\
& \begin{array}{ll}
\mathrm{F}_{3} \mathrm{C} & \mathrm{F}_{3} \mathrm{C}
\end{array}
\end{aligned}
$$

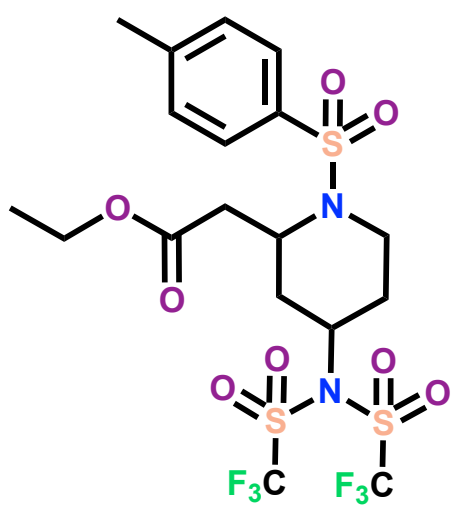




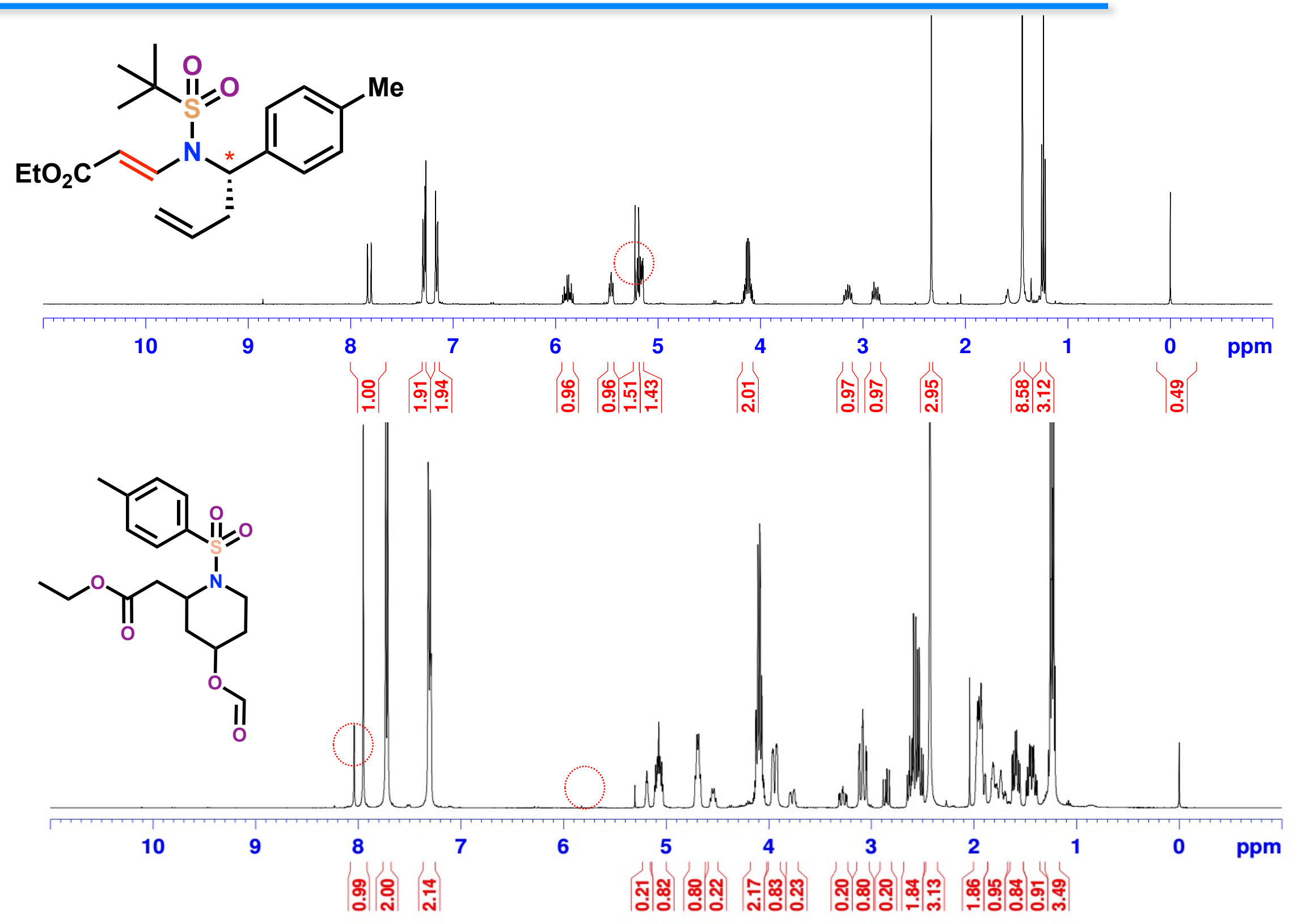




\section{aza-Prins-Ritter}

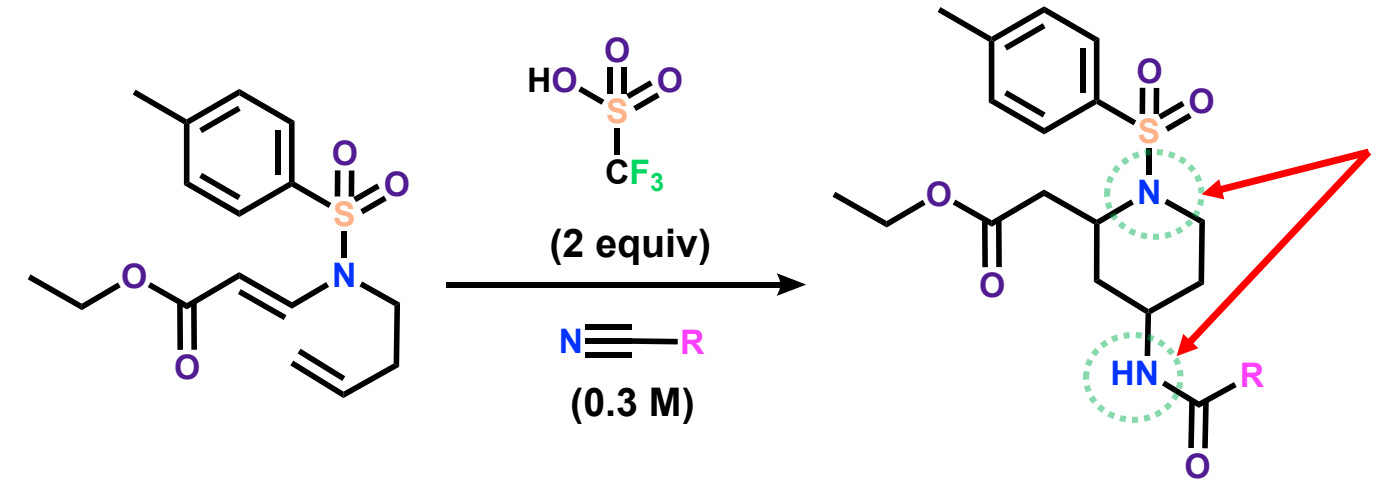

Sites for further modulation!

\section{aza-Prins-Ritter Derivative Library}

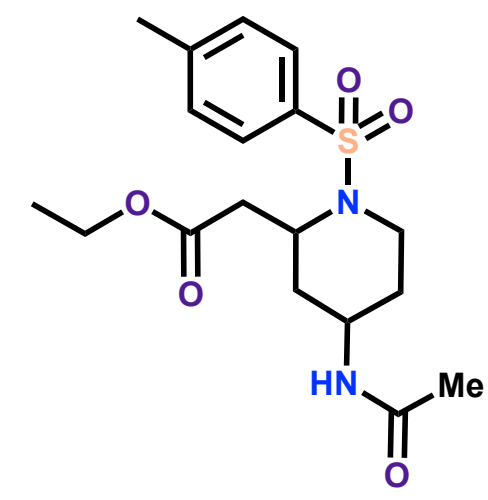

GJR-7-001

$96 \%$

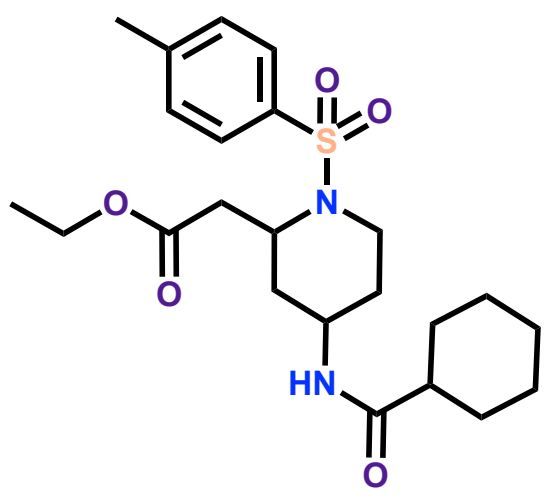

GJR-7-014

$63 \%$

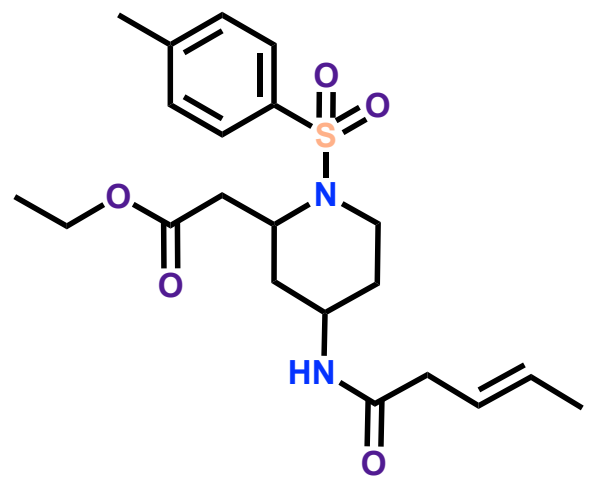

GJR-7-016

$60 \%$

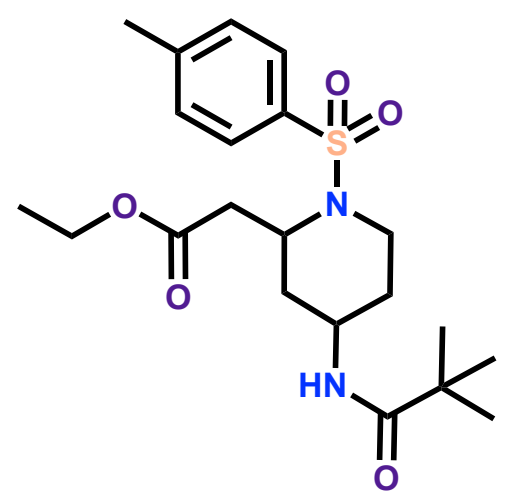

GJR-7-038

$61 \%$ 


\section{Acknowledgements}

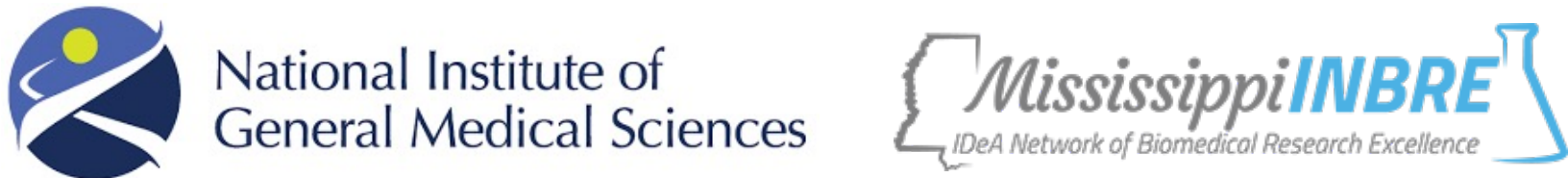
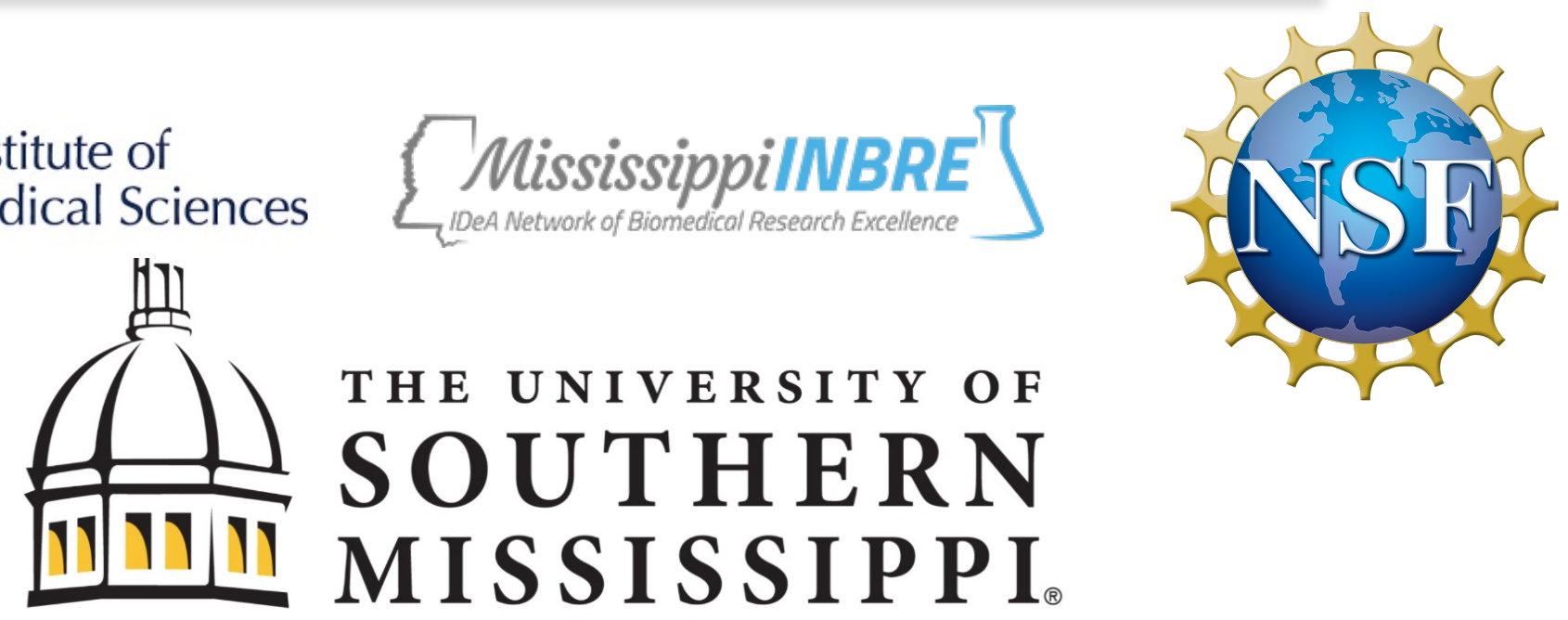

USM College of Arts and Sciences School of Mathematics and Natural Sciences

- Donahue Research Group

Dr. Matthew G. Donahue

Emily Crull

Gavin Rustin

Ana Gilbert
Kaitlyn Birkhoff

Clara Ellis

John A. Hood 